\title{
WHEN IS THE FREE PRODUCT OF LATTICES COMPLETE?
}

\author{
GEORGE GRÄTZER AND DAVID KELLY ${ }^{1}$
}

\begin{abstract}
Yu. I. Sorkin proved that, up to isomorphism, there are three finite lattices that can be represented as a free product of two lattices. In this note we prove that, up to isomorphism, there are five complete lattices that can be so represented.
\end{abstract}

1. Let $A * B$ denote the free product of the lattices $A$ and $B$, and let $C_{n}$ denote the $n$-element chain.

THEOREM. $A$ free product $L=A * B$ of two lattices is complete iff $L$ is isomorphic to one of the following five lattices: $C_{1} * C_{n}, n=1,2,3,4$, and $C_{2} * C_{2}$. In fact, up to isomorphism, $\{A, B\}$ is one of $\left\{C_{1}, C_{n}\right\}, n=1,2,3,4$, or $A \cong B \cong C_{2}$.

This result generalizes a theorem of Yu. I. Sorkin [5], describing when $L=A * B$ is finite; we get his result by deleting the infinite lattices $C_{1} * C_{4}$ and $C_{2} * C_{2}$ from the above list.

2. The "if" part of the Theorem is implicit in a paper of H. L. Rolf [4] where $C_{1} * C_{4}$ and $C_{2} * C_{2}$ are described by (generalized) diagrams. (These diagrams are reproduced in G. Grätzer [1].) A simple inspection of these diagrams shows that $C_{1} * C_{4}$ and $C_{2} * C_{2}$ are (infinite and) complete. $C_{1} * C_{n}$, $n=1,2,3$, are finite, hence complete, by Yu. I. Sorkin [5]. (For their diagrams, see also G. Grätzer [1].)

3. We need some simple facts about isotone retracts of lattices.

Let $L$ be a lattice and let $K$ be a sublattice of $L$. A map $\varphi: L \rightarrow K$ is a retraction (and $K$ is a retract of $L$ ) iff $x \varphi=x$ for all $x \in K$. If $\varphi$ is also an isotone map, then $\varphi$ is called an isotone retraction and $K$ an isotone retract of $L$.

The next two results are trivial and well known.

LEMMA 1. An isotone retract of a complete lattice is complete.

LEMMA 2. Every finite sublattice of a lattice is an isotone retract.

4. Let $L$ be a free product of the lattices $A$ and $B$; we assume that $A$ and $B$ are sublattices of $L$. Let $L^{b}$ denote $L$ with new bounds $0^{b}$ and $1^{b}$ added to $L$.

Received by the editors September 24, 1976.

AMS (MOS) subject classifications (1970). Primary 06A20.

Key words and phrases. Lattice, free product, complete.

'The research of both authors is supported by the National Research Council of Canada.

o American Mathematical Society 1977 
For $x \in L,(x] \cap A$ is principal or $\varnothing$; we define $x_{A} \in A$ by $\left(x_{A}\right]=(x] \cap A$ in the first case and $x_{A}=0^{b}$ in the second case (see G. Grätzer, H. Lakser and C. R. Platt [2] and also G. Grätzer [1]). We define $x_{B}$ similarly, and $x^{A}$, $x^{B}$ dually.

LemMa 3. Let $L=A * B$. Let $A^{\prime}$ be an isotone retract of $A$ and let $B^{\prime}$ be an isotone retract of $B$. Let $L^{\prime}$ denote the sublattice of $L$ generated by $A^{\prime} \cup B^{\prime}$. Then $L^{\prime}$ is an isotone retract of $L$.

Remark. By a result of B. Jónsson [3], $L^{\prime}$ is a free product of $A^{\prime}$ and $B^{\prime}$.

Proof. Let $\alpha: A \rightarrow A^{\prime}$ and $\beta: B \rightarrow B^{\prime}$ be isotone retractions. We define a $\operatorname{map} \lambda: L \rightarrow L^{\prime}:$

For $x \in A, x \lambda=x \alpha$ and for $x \in B, x \lambda=x \beta$; if $x=y \vee z$, then $x \lambda=y \lambda \vee z \lambda \vee x_{A} \vee x_{B}$; if $x=y \wedge z$, define $x \lambda$ dually.

It was proved in G. Grätzer, H. Lakser and C. R. Platt [2], that $\lambda$ is well defined on $L$ and $\lambda$ is isotone. A simple induction shows that $x \lambda \in L^{\prime}$ for all $x \in L$ and that $x \lambda=x$ for all $x \in L^{\prime}$. Thus $\lambda$ establishes that $L^{\prime}$ is an isotone retract of $L$.

5. We need one more observation.

LEMMA 4. Let $L$ be a countable lattice. If $L$ contains $F(3)$ (the free lattice on three generators) as subposet, then $L$ is not complete.

Proof. Since $F(3) \supseteq F\left(\aleph_{0}\right)\left(\mathrm{Ph}\right.$. M. Whitman [6]) and $F\left(\aleph_{0}\right)$ contains the chain of rationals, if $L$ were complete, then $L$ would contain the chain of real numbers, contradicting $|L|=\aleph_{0}$.

6. Now we prove the "only if" part of the Theorem. Let us assume that $L=A * B$ is complete.

If $A$ is not a chain, then $A \supseteq F(2)$. By Lemma 2, F(2) is an isotone retract of $A$, hence by Lemma 3 and the Remark, $F(2) * F(1) \cong F(3)$ is an isotone retract of $L$. By Lemma $1, F(3)$ would be complete, contradicting Lemma 4.

Hence we can assume that both $A$ and $B$ are chains. It was shown in $\mathrm{H}$. L. Rolf [4], that $F(3)$ is isomorphic to a sublattice of $C_{1} * C_{5}$ and also of $C_{2} * C_{3}$. Now if $|A| \geqslant 5$, then $C_{5}$ is an isotone retract of $A$, hence by Lemma $3, C_{1} * C_{5}$ is complete, contradicting Lemma 4 . Thus $|A| \leqslant 4$ and $|B| \leqslant 4$. Now if $|A| \geqslant 3$ and $|B| \geqslant 2$ (or symmetrically), then we proceed as above. Thus either $A \cong B \cong C_{2}$ or $A$ is a singleton and $B$ has at most three elements or symmetrically. This completes the proof of the Theorem.

\section{REFERENCES}

1. G. Grätzer, General lattice theory, Akademie Verlag, Academic Press, Birkhäuser Verlag (to appear).

2. G. Grätzer, H. Lakser and C. R. Platt, Free products of lattices, Fund. Math. 69 (1970), 233-240. 
3. B. Jónsson, Sublattices of a free lattice, Canad. J. Math. 13 (1961), 256-264.

4. H. L. Rolf, The free lattice generated by a set of chains, Pacific J. Math. 8 (1958), 585-595.

5. Yu. I. Sorkin, Free unions of lattices, Mat. Sb. N.S. 30 (72) (1952), 677-694. (Russian).

6. Ph. M. Whitman, Free lattices, Ann. of Math. (2) 42 (1941), 325-330; II (2) 43 (1942), 104-115.

Department of Mathematics, University of Manttoba, Winnipeg, Manitoba, Canada R3T 2N2 